\title{
La antología de Francesco Sansovino y su recepción en España (siglos XVI y XVII)
}

\author{
Francesco Sansovino's anthology of novella \\ and its reception in $16^{\text {th }}$ - and $17^{\text {th }}$ - century Spain
}

\author{
Diana Berruezo Sánchez \\ University of Oxford \\ diana.berruezo-sanchez@mod-langs.ox.ac.uk \\ ORCID iD: http://orcid.org/0000-0003-4629-3614
}

RESUMEN: El presente artículo analiza la exitosa colección de cuentos de Francesco Sansovino atendiendo a su compleja historia textual y a su recepción en España. Por un lado, se detallan las adiciones, supresiones y modificaciones que sufre la antología desde su publicación en 1561 hasta su última reedición en 1610, especialmente por lo que atañe a la carta a los lectores y la posterior identificación del antólogo como autor de las novelas. Por otro lado, se ofrecen datos que atestiguan la circulación del texto en España en los siglos XVI y XVII.

Palabras clave: Francesco Sansovino, novella, Cento novelle scelte, recepción, Lope de Vega, Sebastián Mey, Castillo Solórzano, Juan Pérez de Montalbán.

ABSTRACT: This article delves into Francesco Sansovino's successful anthology of novelle and its complex circulation and reception in Spain. Firstly, it outlines the anthology's corrections, insertions, and deletions from its publication in 1561 to its latest edition in 1610. Secondly, it explains the misinterpretation from which the anthologist is deemed as the author of the short stories. As argued here, the fact that the letter addressed to the readers was not included in the latest editions triggered the misunderstanding about the authorship of the short stories. Finally, it offers significant data that proves the circulation of the anthology in Spain in the $16^{\text {th }}$ and $17^{\text {th }}$ Centuries.

Keywords: Francesco Sansovino, Italian novella, Cento novelle scelte, reception, Lope de Vega, Sebastián Mey, Castillo Solórzano, Juan Pérez de Montalbán. 


\section{INTRODUCCIÓN}

La antología de cuentos de Francesco Sansovino, Cento novelle scelte dai più nobili scrittori, publicada en Venecia en 1561 y reeditada ocho veces hasta 1610, cosechó un éxito notable dentro y fuera de las fronteras italianas. El florilegio, que permitió la circulación de numerosas historias de conocidos novellieri italianos, entre ellos una treintena de cuentos boccaccianos desde la prohibición inquisitorial hasta la versión expurgada del Decamerón, fue una fuente a la que acudieron prosistas y dramaturgos áureos. No obstante, el florilegio no ha sido rigurosamente estudiado por la crítica especializada, debido, en parte, a una compleja historia textual, que todavía no se ha fijado en una edición moderna. De ahí también derivan las imprecisiones críticas respecto a la autoría de las novelas incluidas en la antología. De hecho, algunos estudiosos que buscan fuentes italianas de historias españolas del siglo XVI y XVII atribuyen la autoría de las novelle a Francesco Sansovino, pues se desconoce que fue, en realidad, el antólogo de una exitosa colección de cuentos escritos por Bandello, Boccaccio, Brevio, Masuccio o Parabosco, entre otros. El presente artículo ahonda en la compleja historia textual de la antología, da un argumento plausible que explica el error que persiste en la crítica con respecto a la autoría de los cuentos y ofrece datos significativos que atestiguan la circulación del texto en España durante los siglos XVI y XVII.

\section{LA FORTUNA TEXTUAL DE LAS CENTO NOVELLE SCELTE}

En 1561 Francesco Sansovino, hijo del famoso arquitecto Jacopo Sansovino $^{1}$, publica la primera edición de una entretenida colección de cuentos, Cento novelle scelte dai più nobili scrittori, per Fran. Sansovino. Nelle quali piacevoli et aspri casi d'Amore, et altri notabili avenimenti si leggono. Con privilegio, In Venetia appresso Fran. Sansovino, MDLXI, cuyas ocho ediciones atestiguan un gran éxito editorial hasta 1610. Siguiendo el ejemplo del Deca-

\footnotetext{
${ }^{1}$ Para una biografía sobre Sansovino, puede consultarse el último capítulo de su obra de creación titulada Il Segretario (1579) (Sansovino, 1942), así como la vida que escribió Giorgio Vasari, los datos bibliográficos que aporta Cicogna (1834: 31-91), y los estudios recientes de Di Filippo Bareggi (1988: 16-20), Elena Bonora (1994) y Christina Roaf (1993: 1107-1122). Francesco Sansovino se doctora en leyes en Bolonia, pero desestima su formación y emprende la carrera de literato - entendida en sentido lato- en el momento de mayor auge de los talleres tipográficos en Venecia. Su labor de polígrafo incluye la impresión, edición, corrección, traducción, anotación, divulgación, recopilación e, incluso, la escritura de creación. Cabe destacar la edición fraudulenta de L'ore di ricreatione de L. Guicciardini (1565) a cargo de Francesco Sansovino y que el propio autor trató de enmendar con una edición autorizada según exponen González Ramírez y Resta (2013)
} 
merón, Sansovino reúne un centenar de historias escritas por otros novellieri, las reparte en diez jornadas y las enmarca en un hipotético brote de peste que arrasa la ciudad de Venecia en 1556. Los protagonistas de la antología se recluyen en un locus amoenus para escapar de la plaga y entretenerse con dulces placeres "hor ballando, hor cantando, hor banchettando, e hor festeggiando secondo che tornava lor meglio" (Sansovino, 1561: fol. s.p.).

Las ediciones que se suceden en Venecia hasta principios del siglo XVII no son uniformes, sino que presentan importantes modificaciones en la selección de novelas, el marco de la colección y el paratexto ${ }^{2}$. De hecho, la censura marca la historia textual de la antología no solo por los cambios que sufre el florilegio, sino por las historias compendiadas cuyos autores también figuraron en los índices inquisitoriales. A todas estas cuestiones dedicaremos el presente apartado.

\subsection{Historia del texto}

Las Cento novelle scelte se publican por primera vez en el taller del propio Sansovino, con una dedicatoria firmada en Venecia el 26 de septiembre de 1560 , tiempo antes de la editio princeps de 1561. Esta primera edición incluye una carta dirigida a los lectores, varias ilustraciones al comienzo de las jornadas y una tabla de argumentos al final de volumen. Un año más tarde, el propio antólogo amplía, revisa y publica nuevamente las Cento novelle scelte ${ }^{3}$. En este caso, el paratexto es el mismo que en la princeps, a excepción de la carta laudatoria inicial que, en esta ocasión, se dirige "Al clarissimo M. Sigismondo de'Cavalli. Eletto Oratore al Sereniss. Duca di Savoia” (Sansovino, 1562: fol. 2r).

En 1563 se da a la estampa la tercera edición, a cargo de Rampazetto y fechada el 10 de junio de ese mismo año, donde se conserva el paratexto y se altera la selección de novelas respecto a ediciones anteriores. La cuarta edición, que ve la luz en 1566, mantiene el paratexto e incluye ilustraciones para cada una de las novelas, según reza el título: Cento novelle scelte da più nobili scrittori della lingua volgare di Francesco Sansovino. Nelle quali piacevoli, et aspri casi d'amore, et altri notabili avvenimenti si contengono.

\footnotetext{
${ }^{2}$ La bibliografía final del artículo contiene las signaturas de los ejemplares de cada una de las ediciones consultados para este trabajo, que se han contrastado con la información ofrecida por Passano (1965 [1878]: 541-546).

${ }^{3}$ El título de la segunda edición ya da cuenta de las modificaciones: Le cento novelle scelte da più nobili scrittori della lingua volgare di Francesco Sansovino. Nelle quali piacevoli et aspri casi d'Amore, et altri notabili avvenimenti si leggono. Di nuovo ampliate, riformate, rivedute et corrette per il medesimo. Con privilegio per Anni XV. IN VENETIA MDLXII.
} 
Di nuovo ampliate, reformate, rivedute, et corrette, per il medesimo, et aggiontovi di nuovo le figure in principio d'ogni Novella. Con privilegio per anni xv. In Venetia, MDLXVI.

Cabe destacar que esta edición reordena las novelas de forma distinta e incorpora cuentos de Bandello y Boccaccio sin mencionarlo explícitamente, como se expondrá más adelante.

En 1571 se imprime otra edición, esta vez con un nuevo título ${ }^{4}$, en las prensas de Marchiò Sessa, las mismas que obtuvieron la licencia de impresión de Il Novellino de Masuccio Salernitano a partir de 1531. Esta quinta edición es especialmente interesante por los cambios que presenta. En primer lugar, desaparece el nombre del antólogo en la portada, quizás como respuesta de renovación editorial o, más plausiblemente, para evitar la prohibición inquisitorial, aunque la obra todavía no se hubiera censurado ${ }^{5}$. En segundo lugar, el paratexto mantiene elementos de ediciones precedentes (loa a Sigismondo Cavalli, carta a los lectores, ilustraciones de las novelas y tabla de argumentos al final) y añade un "discorso fatto sopra il Decamerone", donde se examinan algunos aspectos de la obra boccacciana, como el título, el estilo, la intención del autor, el número y orden de las jornadas, y el arte de las novelas ${ }^{6}$. Por último, se incluye, al final del volumen, la primera colección de cuentos italiana, Le cento novelle antiche o Novellino, dedicadas "al reverendissimo monsig. Goro Gherio Vescovo di Fano et digniss. Vicelegato di Bologna, Sig. Et benefattor suo singolariss", y firmadas por Carlo Gualteruzzi, el primer editor de la colección (Bolonia, 1525) $)^{7}$. De hecho, el florilegio de Sansovino reproduce la princeps de Gualteruzzi a quien, según expone en la carta introductoria, le había llegado a las

\footnotetext{
${ }^{4}$ Cento novelle scelte da più nobili scrittori della lingua volgare, con l'aggiunta di cento altre novelle antiche non pur belle per inventione, ma molto vili per l'eleganti et toscae elocutioni necessarie a chi vuol regolatamente scrivere nella nostra lingua. Nelle quali piacevoli, et aspri casi d'Amore, et altri notabili avvenimenti si contengono. Con gli argomenti a ciascuna novella per ammaestramento de'Lettori al viver bene. Et con le figure poste et apropriate a suoi luoghi. Di nuovo rifecute, corrette et riformate in questa Quarta Impressione. Con privilegio, MDLXXI, In Venecia, aprresso gli Heredi di Marchiò Sessa.

5 Para esta cuestión, véase Berruezo Sánchez (en prensa).

${ }^{6}$ Este discurso también añade un interesante comentario para probar la entidad de las novelas. De ahí que Ordine (2009: 183-188) lo haya editado modernamente en una obra de conjunto sobre teoría de la novela.

7 Véase Bottasso (1989: 259) para la fortuna editorial de esta colección. La princeps de las Cento novelle antiche, publicada en Bolonia en 1525, corre a cargo de Carlo Gualteruzzi. Más tarde, circula en un opúsculo sin fecha; en 1571 se incluye en la antología de Sansovino respetando la princeps de 1525; y se vuelven a imprimir en 1572, a cargo de Vincenzo Borghini: Libro di novelle e di bel parlar gentile, nel qual si contengono cento novelle volta mandate da messer Carlo Gualteruzzi da Fano di nuovo ricorrette con aggiunta di quattro altre nel fine e con una dichiarazione d'alcune delle voci più antiche, in Fiorenza, nella stamperia dei Giunti, 1572.
} 
manos esta colección de cien novelas, escrita en vulgar, sin nombre de autor y sin título ${ }^{8}$.

En 1598, con privilegio de 1597, se publica nuevamente el florilegio a cargo de Marco Claseri. En esta ocasión, la edición, dedicada a Annibal Chieppi, presenta el nombre de Sansovino en la portada ${ }^{9}$ y varias modificaciones respecto a la princeps: nuevo corpus y reordenación de los cuentos, omisión de la carta a los lectores y supresión de varios de los argumentos que encabezan las novelas. Asimismo, en esta sexta edición del florilegio, menos cuidada, abundan las confusiones, por ejemplo, en los nombres de los protagonistas, como sucede con Gismondo/Guilio para designar al mismo personaje en la primera jornada, novela segunda.

A principios del siglo XVII, la colección sansoviniana ve la luz dos veces en la imprenta de Alessandro Vecchi, en 1603 y en 1610. Esta última parece ser una reedición de la de 1603, pues ambas conservan una misma dedicatoria a Girolamo Rosetti con rúbrica en Venecia el 10 de mayo de 1603, prescinden de la carta a los lectores y suprimen el marco de la ficción narrativa. Como novedad, la edición de 1603 incorpora marginalia con las enseñanzas y moralejas de las novelas, así como versos instructivos al final de los cuentos. La moraleja final, que tiene precedentes en la tradición de la novella italiana ${ }^{10}$, sintetiza la moraleja de la historia narrada. Un ejemplo de ello se encuentra al final de una novela decameroniana, la de Andreuccio da Perugia:

Come regger si debba Huom; pria che credi

Et feminili inganni, al pianto, e al riso,

Lettore attendi sì, che tu non riedi

Qual Andreuccio Povero, e deriso (Sansovino, 1603: fol.11).

\footnotetext{
${ }^{8}$ Los ejemplares de la quinta edición del florilegio sansoviniano que he consultado presentan importantes diferencias entre sí con respecto a las Cento novelle antiche. El primero de ellos, el ejemplar III.2.69 de la Biblioteca Central Nacional de Florencia (BNCF), contiene ochenta y nueve novelas; el segundo (BNCF, 2.9.5.28) recoge los cien cuentos antiguos. Asimismo, la ficha bibliográfica de un tercer ejemplar en la BNCF (Misc. 1021. 11) indica que se conservan sueltas las Cento novelle antiche: "Ex Legati. [Detta libreria] del C[¿?]. Anton. Francesco [Marmi/?]". Es decir, esta primera colección italiana de novelas circuló independiente de las Cento novelle scelte de Sansovino, aunque se publicaran conjuntamente en 1571. Posteriormente, se asoció a la Libraria o los Marmi de Anton Francesco Doni, por error o por algún motivo que desconozco.

${ }^{9}$ El título de la edición da una aproximación a los cambios paratextuales: Cento novelle scelte da più nobili scrittori della lingua volgare de Franscesco Sansovino, nelle quali piacevoli et notabili avvenimenti si contengono. Di nuovo reformate, rivedute, et corrette, con licentia de' superiori et aggiuntovi le Figure in principio d'ogni Novella. All'Illustriss. Sig. Annibal Chieppi, consigliero del serenissimo Duca di Mantova. Con privilegio. In Venetia, MDXCVII, Appresso Alessandro de Vecchi; y el colofón: "In Venetia, Alla stampa di Marco Claseri. A istanza d'Allessandro di Vecchi, 1598".

${ }^{10}$ De hecho, se trata de un procedimiento análogo al de textos españoles medievales de prosa didáctico-moralizante, como El Conde Lucanor o el Libro de los enxemplos por abc. En este caso, el antólogo tenía modelos narrativos próximos, como la colección de Straparola.
} 
Finalmente, existe una edición de 1619, reseñada por Passano sin más detalles que los siguientes: "-Le stesse. Venetia, 1619. In-4". Figo. Edizione bruttisima" (Passano, 1965 [1878]: 546). Se trata de una edición, quizás fantasma, que no he podido localizar en ningún catálogo ni he visto descrita en otro estudio.

\subsection{El marco narrativo}

La ficción narrativa del florilegio sigue la estela del Decamerón. De ahí que la antología esté dividida en diez jornadas, describa a diez narradores y se sitúe en un locus amoenus propicio para resguardarse de la peste y relatar cuentos entretenidos. El propio Sansovino, al inicio de la antología, justifica su vinculación con el modelo decameroniano ${ }^{11}$, arguyendo que el orden de sus cuentos sigue "quel del Boccaccio, sì perché quello è bellissimo, et sì ancho perché a me pare che sia molto convenevole et facile a questa materia" (Sansovino, 1561: s. p.). Asimismo, la figura del rey o reina de las jornadas sansovinianas, que en este caso no eligen el tema del día, también cumplen funciones análogas al Decamerón, como decidir el orden de los cuentos, distribuir las actividades y despertar al resto de los narradores para empezar el día.

La ficción del marco empieza con la iniciativa de Lucio, uno de los jóvenes narradores que, frente a la pestilente epidemia, reúne a un grupo de amigos en casa, el refugio bucólico donde serán recibidos con apetitosas viandas. El florilegio introduce a los diez narradores de la ficción del marco (Irene, Costanza, Lavina, Lietta y Lisa; Filone, Perotto, Filandro, Lucio y Sisimbo) cuyas características se asemejan a los narradores del Decamerón: "giovani tutti, et di belle lettere, et di gentili et nobili costumi ornati". Como se observa, aunque el desarrollo del marco discurre en paralelo al modelo, en el caso sansoviniano hay cinco hombres y cinco mujeres frente a las siete damas y tres jóvenes boccaccianos. Lietta da el pistoletazo de salida al sugerir que se cuenten historias para amenizar la reclusión; propuesta que secundan los de-

\footnotetext{
${ }^{11}$ La predilección de Sansovino por Boccaccio se aprecia también en el interés que mostró por editar algunos de sus textos. Sansovino edita el Filocolo en 1551 (reeditado en 1554, 1564, 1575, 1612); y el Ameto en 1555 (reeditado en 1558, y póstumamente en 1586 y 1592). En este caso, Sansovino ofrece un comentario final defendiendo la supremacía de Boccaccio frente a Castiglione. Asimismo, el polígrafo veneciano edita el Decamerón y lo publica en las prensas de Gabriele Giolito en 1546 (reeditado en 1548, 1550 y 1552) (Bonora, 1994: 48). Además, Sansovino escribe una biografía del poeta de Certaldo que se publica en una edición veneciana del Decamerón, en los talleres de Vincenzo Valgrisio, en 1554. Por si fuera poco, cabe sumar una obra de creación, las Lettere sopra le dicei giornate del Decamerone (cfr. Bonora, 1994: 79-96 y Roaf, 1993: 11071121), donde se incluyen cien cartas (en realidad 107) relacionadas con las cien novelas de Boccaccio.
} 
más narradores y que, tras el orden establecido por Sisimbo, da lugar a la inserción de las cien historias.

El orden de las novelas sigue el imaginario de cualquier lector de novelle: el del Decamerón. De ahí que se otorgue a Lietta el título de reina de la primera jornada simbolizado en la corona de laurel. La nueva reina, después de cenar y pasear por los lujosos jardines del locus amoenus, pide a los demás narradores "di ragionar cose dilettevoli et belle" para el día siguiente. Además de contar historias, las otras actividades de los narradores recuerdan a las labores del Decamerón: van a misa por las mañanas, pasean, se solazan, pasan la regencia con la corona de laurel, y terminan los días con placenteras canciones.

Como sucedía en el original boccacciano, las novelas están divididas en varias secciones (argumento, parlamento del narrador y cuento), aunque la colección de Sansovino ofrece, además, una alegoría o moraleja, ausente en el Decamerón. El siguiente ejemplo, perteneciente a una novela de Boccaccio incluida en la antología de $1571(\mathrm{~V}, 7)$, es ilustrativo de ello:

Il proposto di Fiesole ama una donna vedova, non è amato da lei, et credendosi giacere con lei, giace con una sua fante, et i fratelli della donna vel fanno trovare al Vescovo. Novella VII.

[ilustración]

Nel che si dimostra che gli huomini di qualche riputatione debbono schivarsi da commetter cosa che macchi l'honor loro (Sansovino, 1571: fol. 111v).

Asimismo, el vínculo del florilegio con el marco decameroniano pasa por la elección del tema de las jornadas, la estructura de las novelas, la reacción de los personajes frente a las historias narradas, las baladas al final de las jornadas, y, también, por un detalle revelador en una de las novelas contenidas en la antología. Se trata del cuento XLVIII de Masuccio, recogido, con sucesivos cambios, en las tres primeras ediciones del florilegio. Una de las variantes que incorpora Sansovino es la sustitución de un pasaje donde Masuccio describía detalladamente la alegría del rey de Túnez al encontrarse con su hijo Malem:

El re de Tunisi che gran parte del ponente avea da Cristiani renegati e da altra gente fatto el suo figliolo con diligentia cercare, né in alcun lato mai niuna nova sentitone, vedendoselo sì ben vestito e onorato davanti, oltra l'amore e la carità paterna, quanto la speranza di lui era del tutto mancata, tanto fu la soa contentezza e la dimostrata festa maggiore, e doppo le infinite accoglienze, d'ogni suo passato accidente fatto, mandò per utto el suo dominio ché del recuperato suo Malem grandissima dimostratione de allegrezza ciascuno facesse; e così fu fatto (Masuccio, 2000: 541).

El mismo pasaje se simplifica en el florilegio, y Sansovino exhorta a la imaginación de las lectoras $-\mathrm{y}$ no lectores - para que recreen el júbilo del 
rey: "vedendoselo venir innanzi così en vestito et furo de ogni sua speranza ritrovato, se fu allegro pensatelo voi medesime" (Sansovino, 1561: fol. 316v$317 \mathrm{r}$ ). Es decir, el antólogo interpela a un público femenino, el mismo que se describe en el proemio decameroniano; un público, no obstante, que no aparece en ningún otro lugar de la antología. Seguramente, Sansovino tenía en mente el modelo que él mismo había editado con anterioridad, el Decamerón, de la misma manera que editores, lectores y antólogos proyectaban un auditorio femenino como receptor indiscutible de las colecciones de cuentos, según había consolidado el modelo de Boccaccio.

Por otro lado, cabe destacar que el marco narrativo de Sansovino presenta numerosas divergencias en las distintas ediciones, ya sea alterando, enmendando, suprimiendo o añadiendo información. Por ejemplo, el lugar de reclusión del marco en la primera edición de la antología es la localidad de Treville ${ }^{12}$ mientras que pasa a ser Oriago $^{13}$ en las demás ediciones con marco (1562, $1563,1566,1571$ y 1598).

Otro de los cambios en la ficción del marco narrativo se halla en la introducción a las jornadas, dedicada, por lo general, a presentar las actividades de los narradores antes de la caída de la tarde, momento en que empiezan sus relatos. Dichas introducciones difieren en las sucesivas ediciones de la antología: se suprimen fragmentos ${ }^{14}$, se desplazan pasajes de una jornada a $\operatorname{otra}^{15} \mathrm{y}$ se introducen variantes en un mismo pasaje ${ }^{16}$.

12 "Treville, luogo sul Trevigiano amenissimo, et forse il piu bello che habbia la Marca, percioch'egli oltre alla comodità per rispetto di Castel Franco che gli è poco lontano, e così fattamente adornato di ricchi et begli arnesi, che non si puo desiderare piu oltre, conciosa che dilettandosi di questo luogo il suo Signore, che fu M. Federigo de Priuli, spendendovi larghi tesori, lo havea fatto stanza piu tosto Imperial ch'altramente. In questo luogo adunque essendossi costoro fermati con le lor donne, cominciarono a darsi insieme il piu bel tempo del mondo hor ballando, hor cantando, hor banchettando, e hor festeggiando secondo che tornava lor meglio" (Sansovino, 1561: s. p.).

13 “'Oriago luogo sul Padovano amenissimo, et bello, percioch'egli, oltre alla comodità per rispetto di Venegia et di Padova che gli è poco lontano, è così fattamente adornato di richi et belli arnesi, che non si puo desiderar piu oltre. In questo luogo aunque essendossi costoro fermati con le lor donne in casa della buona memorea di M. Francesco Lando, et raccolti da M. Nicolò Manolesso gentilhuomo ottimo, et da M. Giovanni Lando con tutte quelle maggiori accolgienze che piu si potevano, cominciarono a darsi insieme il piu bel tempo del mondo, hor ballando, hor cantando, hor banchettando, e hor festeggiando secondo che tornava lor meglio" (Sansovino, 1562: s. p.).

14 Por ejemplo, el pasaje introductorio a la cuarta jornada de la edición de 1561 ("Era già l'oriente tutto bianco [...] e così disse") se omite en las ediciones de 1566, 1571 y 1598.

${ }^{15}$ El siguiente pasaje pertenece a la segunda jornada de la princeps, se reproduce igualmente en 1571, pero se traslada a la tercera jornada en las ediciones de 1566 y 1598: "Poi che'l Sole indorando co suoi raggi i vicini colli, diede segno a mortali del sopravegnente giorno, Perotto ch'era piacevolissimo giovane levatosi cominciò scherzevolmente a chiamar sù sutti gli altri, riprendendogli piacevolmente che stessero tanto nel letto, perché levato sù il Re et fatti tuti gli altri levare, diede ordine che s'udisse la messa, dopo la quale havendo ragionatao assai, desinarono allegramente, et si riposarono parte di loro secondo il costume, et parte giocarono un pezzo. Ma 
El cambio más significativo se halla en la omisión completa del marco en las ediciones de 1603 y 1610. El florilegio, que empieza directamente con la narración de los cuentos, prescinde del prólogo donde se describe la plaga bubónica en Venecia y las acciones que emprenden los narradores. Ahora bien, las huellas del marco se pueden rastrear en ambas ediciones, sobre todo al inicio y final de las novelas, donde encontramos párrafos en los que se describen las reacciones de los narradores, la entrega de la corona de laurel al rey de la siguiente jornada, o las baladas finales.

Cabe pensar que estos vestigios son el resultado de la labor de impresores y tipógrafos, pues la desaparición de los párrafos iniciales (donde se escucha a los narradores) hubiera conllevado una nueva y compleja distribución de los folios, mientras que la supresión de los primeros folios, donde se describe el marco de la ficción narrativa, resultaba más fácil ${ }^{17}$. Como consecuencia de ello, las últimas ediciones de la antología presentan una clara contradicción: sabemos que las historias las cuentan narradores de los que, sin embargo, no poseemos información alguna. Claro está que el horizonte de expectativas de los lectores podía complementar la ausencia de información, pues la mayoría de colecciones de cuentos presentaban una misma estructura. Es decir, la descripción del marco narrativo podía resultar incluso redundante, y eso facilitaba el ahorro de papel a los impresores de la época.

En esta línea, no es ocioso recordar que los imitadores de Boccaccio fueron adecuando los marcos narrativos de las colecciones de cuentos a la demanda

poiché al Re piacque che ogniuno si adunassi nel luogo usato, posto ciascuno a sedere come ben gi venne, il Re impose a Lietta che desse principio alle novelle della presente giornata, la quale percioche baldanzosa era così comincio a dire" (Sansovino, 1561: fol. 57r-57v).

${ }^{16}$ El pasaje citado en la nota anterior presenta variantes respecto a la princeps, especialmente en la edición de 1598, como puede verse a continuación. Se han tachado las omisiones de 1598 y se han escrito en cursiva las modificaciones y añadiduras. "Poi che'l Sole indorando con suoi raggi i vicini colli, diede segno à mortali del sopravegnente giorno, Perotto ch'era piacevolissimo giovane levatosi cominciò scherzevolmente a chiamar sù tutti gli altri, riprendendogli che stessero tanto nel letto, perché levata sù la Reina et fatti tutti gli altri levare, diede ordine che s'udisse la messa, dopò la quale havendo ragionato assai, desinarono allegramente, et elsi riposarono secondo il costume, et parte giocarono un pezzo. Ma poiché alla Regina piacque, che ogniuno si riducesse nel luogo usato, posto ciascuno a sedere come ben gli venne, la Reina impose a Lietta che desse principio alle novelle della presente giornata, la quale percioche baldanzosa era così cominciò a dire" (Sansovino, 1598: fol. 73-74).

17 Un proceso análogo lo encontramos en la edición semiclandestina de Il Novellino de Masuccio Salernitano, conocida como La Gatta y publicada después de 1565. La crítica especializada ha señalado reiteradamente que los cortes en esta edición responden a los expurgos censorios, pues la obra del salernitano entró en los primeros índices inquisitoriales (1557-1559). Sin embargo, como han analizado Terrusi (1998: 270-271) y Pirovano (1996: 396-397), La Gatta omite solamente los exordios y conserva los fragmentos obscenos y anticlericales que le hubieran valido la censura. El recorte de las partes menos importantes de la obra se deben, por lo tanto, a motivos comerciales, como el ahorro de papel. 
comercial de la época ${ }^{18}$. Los lectores mostraron mayor predilección por las historia y desestimaron, paulatinamente, la arquitectura que las envolvía. De ahí que la simplificación —o incluso supresión — del marco fuera la opción predilecta de escritores, editores e impresores en el siglo XVI. En el caso de las Cento novelle scelte, queda claro que Sansovino, pese a vincularse a Boccaccio, no llega a explotar la potencialidad narrativa del marco decameroniano, hasta el punto que las dos últimas ediciones lo omiten parcialmente, quizás para acomodarse a los intereses de los lectores. Cabe precisar, no obstante, que las ediciones de 1603 y 1610 son póstumas, lo que permite especular que en vida, quizás, la fidelidad de Sansovino al modelo boccacciano no hubiera permitido tales mutilaciones textuales.

En cualquier caso, las modificaciones señaladas aquí atestiguan el éxito de un producto editorial —una antología de novelle - que circuló profusamente, y se adaptó a las exigencias de lectores, editores e impresores de los siglos XVI y XVII.

\subsection{La lista de escritores en el proemio}

Francesco Sansovino abre la antología de cuentos con una epístola a los lectores. En ella, ofrece la nómina de novellieri cuyas historias ha incluido en el florilegio, aunque sin detallar la novela exacta seleccionada para la ocasión. La lista de escritores seleccionados incluye a Straparola, Parabosco, Firenzuola, Gratia, Erasto, Brevio, Molza, Ser Giovanni Fiorentino, Masuccio Salernitano, y alguna novela del propio Sansovino, "le quali sono state da me raffettate et racconcie nella lingua per quanto io ho potuto, et secondo che dalla fretta de gli Stampatori m'è stato conceduto" (Sansovino, 1561: fol. 4v).

A pesar de los cambios que se suceden a lo largo de las ocho ediciones del florilegio -incluyendo variaciones en las novelas y autores seleccionados-, la carta a los lectores se conserva íntegra en las ediciones de 1561, $1562,1563,1566$ y 1571 . Es decir, la nómina de nobili scrittori permanece intacta. Sin embargo, el análisis de las ediciones muestra cómo el número de historias se altera, ofreciendo como cómputo final de todas las ediciones la cifra de 165 cuentos $-\mathrm{y}$ no los 100 anunciados—, que, además, pertenecen a escritores no mencionados en la lista inicial. A todo ello hay que sumar el hecho de que el proemio desaparece en las tres últimas ediciones (1598, 1603,

${ }^{18}$ Véase Malato (1989: 36-39) y Minutelli (1990: 13) para las distintas estructuras de las colecciones de cuentos, así como las diferencias con el modelo decameroniano; Plaisance (1989: 103-118) para profundizar en las funciones y tipologías de los marcos; y Bottasso (1989: 259-260) para el éxito de las colecciones sin marco de facecias y proverbios. 
1610), quizás para escapar de los índices inquisitoriales, dado que muchos de los novellieri explícitamente nombrados en la carta a los lectores estaban censurados (Berruezo Sánchez, en prensa).

A finales del siglo XIX, Passano elaboró una lista exhaustiva detallando el nombre del escritor al que pertenece cada una de las novelas incluidas en la antología (Passano, 1965 [1878]: 546-553), identificando, además, las novelas que pertenecen a Bandello y Boccaccio. De esta manera, Passano amplía la lista de escritores seleccionados en el florilegio, y nos permite observar cómo a partir de la edición de 1562 contamos con veintiún cuentos de Bandello reproducidos, en su mayoría, en las ulteriores ediciones: tres historias bandellianas desaparecen después de 1571 y otra se omite en 1563 (sustituida, a su vez, por otro cuento bandelliano que solo aparece en esa tercera edición). Además, el florilegio cuenta con otras cuatro novelas de Bandello que, según Passano, comparten atribución con Fiorentino, Parabosco o Firenzuola.

Asimismo, la tabla de Passano da cuenta de las novelas de Boccaccio incluidas a partir de la cuarta edición de la antología (1566) sin que aparezca especificado en el proemio. Del Decamerón se introduce una treintena de historias, aunque cuatro de ellas podrían ser también de Il Pecorone de Fiorentino (dos de estas cuatro novelas están presentes en todas las ediciones, una aparece solo en $1563^{19}$ y la cuarta figura solo a partir de 1566). La inclusión de cuentos boccaccianos en el florilegio es muy significativa para la difusión del Decamerón, pues permite que esta treintena de historias boccaccianas circulen por Italia —y tal vez por España - después de la censura de 1559 y antes del expurgo de 1573.

\section{LA CIRCULACIÓN DE LA ANTOLOGÍA EN ESPAÑA}

El florilegio de Francesco Sansovino es un vehículo importante para la difusión en España de importantes novellieri italianos, como Giovanni Boccaccio, Matteo Bandello o Masuccio Salernitano. No obstante, la crítica no ha dedicado un estudio detallado al respecto, a cuya laguna bibliográfica se ha querido dedicar el presente artículo. Concretamente, en este apartado se quiere mostrar cómo varios escritores áureos leyeron la antología y, quizás, sacaron inspiración para sus obras de creación.

Primero, cabe recordar con Anastasio Rojo Vega que la primera parte del florilegio se tradujo al español por iniciativa del impresor Milis. Dicha empresa, no obstante, no llegó a ver la estampa, pese a que el impresor Godínez de

${ }^{19}$ En nuestra opinión, es una historia del Fiorentino que se inspira en Boccaccio. De otra manera, querría decir que el poeta de Certaldo se habría incluido en el florilegio desde 1563. 
Milis obtuvo la licencia para publicar "la primera parte de las cien novelas escogidas de Francisco Sansobino" (Rojo Vega, 1994: 149) ${ }^{20}$.

Segundo, es importante destacar las menciones al florilegio documentadas por ingenios áureos. Una muestra la encontramos en el prólogo de La ingeniosa Elena (1614) de Salas Barbadillo, concretamente, en la "Carta al lector" firmada por Lugo y Dávila ${ }^{21}$, quien, como se muestra a continuación, menciona a Sansovino. A modo de curiosidad, cabe decir que la "Carta al lector" $-\mathrm{y}$ por tanto la alusión a Sansovino - no figura en la versión anterior de la obra de Salas Barbadillo, La hija de Celestina.

Y assí como en la Antigüedad los griegos, conformándose con la disposición de su siglo, escrivieron en prossa los poemas de Theágenes y Cariclea, Leucipo y Clitofonte, tan llenos de conmiseración en tanta variedad de casos, ya quietando y ya oprimiendo el ánimo en el discurso de sus peregrinaciones hasta dexarlos en prosperidad; y por los latinos (qual se vee en las narraciones amorosas de Plutarco), más breves, se escriven obras semejantes con fines amorossos infelices (como en La Aristoclea y otras); y después los italianos tanta multitud de novelas que las sacaron a la luz de ciento en ciento (no siendo menos los autores desde el Bocacio, que sus nombres, y aun no de todos, hallarás en el proemio del Sansovino), ahora, para conseguir Alonso de Salas el fin que con tales obras se pretende, te muestra en la astucia y hermosura de Elena y trato de su compañía lo que executa la malicia deste tiempo y el fin que tiene la gente desalmada, que viven como si les faltara el conocimiento de nuestra verdadera $\mathrm{Fe}$ y de que ay premio y castigo eterno (Salas Barbadillo, 1985: 41-42).

Otro ejemplo de alusión al polígrafo veneciano se halla en La quinta de Laura de Castillo Solórzano:

Pues para divertir el tiempo, tratò esta dama, de que en conformidad todas sus dama la entretuviessen, yà con musica, yà con danças, y yà con novelar, exercicio mui usado en Italia; diganlo los Vandelos, Sansovinos, y Bocacios, que tantos tomos han impresso dellas, y aora en España los han excedido con grandes ventajas; pues esto se haze con mas primor, y propiedad para entretenimiento de los Lectores, y suspensión suya (Castillo Solórzano, 1649: fol. 3);

\section{y en Las harpías de Madrid (1631):}

\footnotetext{
${ }^{20}$ Dicha información se encuentra en los Libros de relaciones, donde "se anotaban las licencias concedidas por el Consejo para la impresión de libros. Recogen la fecha de la primera licencia o de la prórroga, el nombre del solicitante, que en la mayoría de las veces es el propio autor, y de la descripción o título del manuscrito, según y como le parecía al empleado de la administración central" (Rojo Vega, 1994: 132).

${ }^{21}$ Corrijo aquí una error de mi tesis (Berruezo Sánchez, 2014). Quien cita la antología no es Salas Barbadillo, sino Lugo y Dávila, quien firma el paratexto de la edición. Esta corrección permite avalar, más si cabe, la propuesta de Fernández Rodríguez (2016b: 222-223), según la cual Lugo y Dávila manejó la antología de Sansovino en su Teatro popular.
} 
- Tomé, como digo, un libro de novelas de un italiano llamado Francisco Sansovino, que escribe en su idioma, en el cual leí la altivez y crueldad de una dama francesa con su amante, que fue extraña debiéndole tanto amor y voluntad (Castillo Solórzano, 1985: 170).

En realidad, Castillo Solórzano, como parte de su ficción narrativa, intercala una historia del florilegio que se ha atribuido al mismo Sansovino en ediciones recientes ${ }^{22}$. Se trata de una confusión extendida en la crítica especializada, y fácilmente comprensible a la vista de los dos ejemplos aquí expuestos, donde podemos advertir una diferencia fundamental. La edición de la antología utilizada por Lugo y Dávila debía conservar la carta a los lectores en la que Sansovino ofrece la lista de los escritores seleccionados para la antología; por lo tanto, debía ser una edición anterior a 1598. Recordemos que las tres últimas ediciones del florilegio $(1598,1603$ y 1610) carecen de la epístola inicial. De hecho, Fernández Rodríguez (2016b: 223 y 2016c: 55) concluye que debe tratarse de la edición de 1561, puesto que Lugo y Dávila se inspira en dos relatos, uno de Firenzuola y otro de Parabosco, para crear sendas novelas, "El médico de Cádiz" y "El andrógino", y ambos modelos italianos se recogen en la colección sansoviniana.

Por otro lado, cabe pensar que Castillo Solórzano manejaba una de las postreras ediciones, pues, a tenor de sus palabras, considera que el autor de los cuentos es Sansovino. Es decir, de esta confusión parecen desprenderse las opiniones críticas modernas según las cuales Sansovino es autor de novelas que, en realidad, pertenecen a otros novellieri, como se ha visto ${ }^{23}$.

Asimismo, las confusiones se multiplican en la crítica especializada al identificar las novelas utilizadas por nuestros prosistas, debido a la compleja historia textual de la antología. Un ejemplo notorio es el caso de la novela "El pronóstico cumplido" de Castillo Solórzano. Según Helí Hernández (1982: 47) — que cita a Bourland ${ }^{24}$ _, "la novela 4 -El pronóstico cumplido- de las Noches entretenidas de Castillo Solórzano, tiene su fuente en la novela 4 de la Séptima Jornada de las Cento Novelle de Sansovino". En realidad, Castillo Solórzano escribe varias colecciones de cuentos, entre ellas Tardes entretenidas (a la que pertenece dicha novela) y Noches de placer - y no "Noches entretenidas"- . Además, Fernández Rodríguez (2016b: 224, nota 30 y 31) ha

22 "F. Sansovino: novelista italiano del siglo XVI, autor de, entre otras cosas, Cien novelas" (Jauralde Pou, 1985: 170). Bourland (1973 [1927]: 59) señala que se trata de la décima novela de la jornada sexta. No obstante, tras revisar las ediciones de la antología, advertimos que esa numeración no se corresponde con la historia intercalada por Castillo Solórzano.

${ }^{23}$ Afortunadamente, Giuliani (1992: 35) ya ha apuntado esta confusión con una de las novelas que pertenece a Brevio y que críticos anteriores, como Profeti, habían atribuido a Sansovino.

24 "Castillo Solórzano, El pronóstico cumplido (Noches, nov. 4), whose original is [Sansovino], Cento novelle, Giorn. 7, nov. 4" (Bourland, 1973 [1927]: 59). 
detectado un error de mi tesis doctoral (Berruezo Sánchez, 2014) al atribuir la novela a Brevio, pues, en realidad, corresponde a un cuento de Erasto. Según corrige Fernández Rodríguez, la novela imitada por Castillo Solórzano aparece en la posición indicada por Bourland solo en 1566, pero en otras posiciones en las demás ediciones.

Un tercer dato que acaba de confirmar la difusión del florilegio en nuestra Península es la inspiración que proporcionó a varios ingenios áureos. Por ejemplo, Luis Gaitán de Vozmediano, el traductor al español de la colección de cuentos de Giraldi Cinthio, los Ecatommiti, afirma en el prólogo que ha eliminado la segunda novela de la primera década y que en su lugar ha puesto "la del maestro que enseña a amar, tomada de las ciento que recopiló el Sansovino"25. En realidad, se trata de un cuento de Ser Giovanni Fiorentino, el autor del Pecorone (escrito hacia 1378 y publicado por primera vez en 1558), que lleva por título "Bocuiuolo romano facendosi insegnare l'arte d'amore". El Proyecto Boscán (catálogo sobre las traducciones de obras italianas al español), además de describir las diferencias entre la novela italiana y la traducción española, afirma que Gaitán de Vozmediano debió de manejar la edición de 1563 del florilegio sansoviniano.

Por otro lado, Sebastián Mey también utiliza varios cuentos de la antología de Sansovino para componer su Fabulario (1613). Por ejemplo, la fábula LV, "El médico y la mujer" (Mey, 1975: 171-175), traduce/adapta el siguiente cuento de la antología (se trata de una historia que pertenece, en realidad, a las Cento novelle antiche):

Maestro Antonio Gerualla medico prende moglie, ella gli fa una fanciulla in due mesi, e il medico con pronta proposta rimandatala a casa se ne liber ${ }^{26}$.

A principios del siglo XX, Buchanan (1906a: 170) ya indicó que la fábula XXVIII de Mey, "El hombre verdadero y el mentiroso" (Mey, 1975: 65-75), procedía de una novela de Firenzuola, actualizada posteriormente por Doni. A la información aportada por Buchanan, hay que añadir ahora que el cuento de Firenzuola se incluye en el florilegio de Sansonvino (Passano, 1965 [1878]: 548), desde donde, quizás, la pudo leer Sebastián Mey:

\footnotetext{
${ }^{25}$ Cita tomada de la edición que hace González Ramírez (2012: 824) de los paratextos de la traducción de Vozmediano. El mismo autor ya había citado este pasaje anteriormente (González Ramírez, 2011: 1233), aunque sin especificar el novelliere al que pertenece la novela sustituida por Gaitán de Vozmediano. Para ello, véase el Catálogo Boscán (Muñiz, 2002-2009) en la entrada de Giovanni Fiorentino.

${ }^{26}$ Sansovino (1561: X, 4). Buchanan (1906b: 204) y Bravo-Villasante (1975: 21) sitúan la novela en la cuarta jornada, segunda novela, de la antología sansoviniana. No obstante, no indican la edición que leyó Sebastián Mey, y, en realidad, la novela pertenece a distintas jornadas según la edición que se maneje: 1561 (X, 4); 1562 y 1563 (II, 8); 1566, 1571, 1598, 1603 y 1610 (IV, 2).
} 
Due compagni truovano un Tesoro, l'uno d'essi lo ruba, vanno al Podestà, et sopara il luogo si conosce la fraude del ladro, il quale dal giudice recive la debita pena (Sansovino, 1561: III, 8) ${ }^{27}$.

Asimismo, la última novela de Masuccio Salernitano se corresponde con la fábula XLIX de Sebastián Mey, "El caballero leal a su señor" (Mey, 1975: 138147), según indicaba ya Buchanan en su artículo. En esta ocasión también cabe añadir que el cuento masucciano aparece en todas las ediciones del florilegio.

Es decir, los datos aquí presentados hacen pensar que Sebastián Mey manejó una o varias ediciones de las Cento novelle scelte. Seguramente, debió de inspirarse en una de las tres primeras ediciones de la antología, pues en ellas se encuentran todas las historias traducidas/imitadas por Mey. Según señala acertadamente Fernández Rodríguez (2016c: 60), que también se ha interesado por la antología de Sansovino como vía por la que circularon las historias de Firenzuola, Mey debió de utilizar la edición de 1561 (Fernández Rodríguez, 2016a, 2016b y 2016c $)^{28}$.

Para constatar la circulación del florilegio por la España de los siglos XVI y XVII es interesante reparar en el siguiente detalle. Sabemos que Juan Pérez de Montalbán recreó una historia de Giovanni Brevio ("Madonna Lisabetta") en la cuarta novela de Sucesos y prodigios de amor (1624), "La mayor confusión", y que esta novella de Brevio se incluye en el florilegio sansoviniano ${ }^{29}$. También sabemos que Pérez de Montalbán dedica su cuento al Fénix, después de que Lope de Vega le hubiera dedicado una comedia:

Al Lope Félix de Vega Carpio, procurador fiscal de la Cámara Apostólica y su notario descrito en el archivo romano.

En la décimatercia parte de sus comedias de v. m. me dedicó una (efecto más de su amor que de mis méritos), y aunque pagar sin ventajas el beneficio pueda llamarse agradecimiento ingrato [...]. Esta novela de La mayor confusión, cuyo caso tiene mucha parte de verdad, restituyo a v. m. como cosa suya, porque si lo poco que he alcanzado en mis pocos años lo debo a su doctrina, a cuyos pechos me he criado siempre, volver al mar lo que salió de su abundancia más se debe llamar restitución que ofrenda. Yo me holgara pareciera de v.m porque, en efeto, fuera de Lope, aunque esto no fuera difícil de creer en muchos que, pensando deslucir algunas obras mías y viéndose

\footnotetext{
${ }^{27}$ Esta novella aparece en las Cento novelle scelte en III, 8 (1561) y V, 4 (1562 y 1563). Véase Passano (1965 [1878]: 548).

${ }^{28}$ Estas investigaciones, aparecidas desde la recepción hasta la publicación del presente artículo, complementan la información aquí presentada.

${ }^{29}$ Bourland (1973 [1927]: 60) señala la antología como fuente de inspiración para Pérez de Montalbán. Además, añade que Margarita de Navarra (Heptamerón, 30) y Bandello (Novelle, II, 35) también recrean este mismo argumento. No obstante, la crítica ha matizado esta aseveración atribuyendo a Brevio la autoría que, en rigor, le pertenece. Véase Dixon (1983: 18) y Giuliani (1992: 18).
} 
convencidos a que están escritas con acierto, se las atribuyen a v. m., error grande de su mala intención, pues no advierten que mejorándolas de dueño las califican, y lo mismo que intentan para desconsolarme viene a servirme de panegírico (Pérez de Montalbán, 1992: 131-132).

La obra del Fénix dedicada a Pérez de Montalbán, La francesilla — “La Francesilla. Comedia famosa de Lope de Vega Carpio. Dirigida al Licenciado Iuan Pérez en la Universidad de Alcalá" (Vega, 1620: fol. 78r)—, es una comedia inspirada en dos cuentos de Masuccio Salernitano. La novella XLV, incluida en todas las ediciones de la antología, inspira el argumento lopesco y la XII da claves para la estructura de la comedia (McGrady, 1981: 7-10 y Berruezo Sánchez, 2013: 750-753). Dada la amistad que trabaron ambos escritores ${ }^{30}$, y a pesar de la voluntad por encubrir las fuentes de inspiración, nos parece plausible pensar que Lope y Pérez de Montalbán intercambiaron lecturas, entre ellas, el florilegio de Sansovino, que sirvió de inspiración literaria a ambos escritores. De ahí, quizás, que Pérez de Montalbán devuelva al Fénix lo que el maestro le había enseñado.

Finalmente, para acabar de enmarcar la difusión de la antología, cabe decir que Lope de Vega utiliza otros tres cuentos de Masuccio Salernitano para su creación dramática (Berruezo Sánchez, 2015: 182-210), todos ellos presentes en el florilegio de Sansovino: El mejor alcalde, el rey (Menéndez Pelayo, 1969: 159 y Berruezo Sánchez, 2012: 144-145) se inspira en el cuento XLVII; Castelvines y Monteses (Krenkel, 1887: 54-55; Gasparetti, 1939: 393 y Berruezo Sánchez, 2012: 142-143) se basa en el XXXIII, a través de la versión de Bandello; y, finalmente, El galán Castrucho y El llegar en ocasión encuentran ecos en el cuento XXIX (Molina, 2002: 1094). Precisamente en El llegar en ocasión confluyen varios textos italianos, entre ellos, un cuento decameroniano (II, 2) que Lope rescribe, y dos cuentecillos de la primera colección de novelas italiana, las Cento novelle antiche, como ha señalado Segre (1990: 105). De acuerdo con lo expuesto más arriba, esta colección manuscrita vio la estampa en 1525, y fue nuevamente impresa por los Sessa en 1571 como apéndice del florilegio de Sansovino (Bottasso, 1989: 259). Quizás Lope pudo manejar una de estas ediciones de la antología donde encontró varios cuentos que inspiraron su producción dramática.

\section{CONCLUSIONES}

Las Cento novelle scelte se caracterizan por una intricada historia textual, marcada, como se ha visto en el presente artículo, por eliminaciones, añadiduras y cambios en las ocho ediciones que se sucedieron en los siglos XVI y XVII.

\footnotetext{
${ }^{30}$ Véase Profeti (1970: 7-32) para la amistad entre Lope y Montalbán.
} 
La consulta de varios ejemplares de cada una de las ediciones nos ha permitido precisar la información proporcionada por Giambattista Passano en el catálogo de 1878, y, además, corregir algún error que la crítica moderna ha repetido en varias ocasiones.

La atribución de las novelas del florilegio a Sansovino es un error arraigado en la crítica moderna que puede deberse a una cuestión catalográfica, pues ha sido entendido como autor por aquellos que se han acercado a obras colectivas o catálogos. Por otro lado, en el siglo XVII, Castillo Solórzano lo consideraba autor de las novelas quizás porque las ediciones que el escritor español manejaba no contaban con la "carta a los lectores": un proemio donde Sansovino enumeraba los escritores de donde había tomado los textos. La corrección de estas y otras imprecisiones repetidas por la crítica especializada, así como la descripción de la antología que aquí se ofrece, permitirá que futuros investigadores interesados en estudiar las Cento novelle scelte como fuente de la literatura española sepan con certeza qué textos contenía, puesto que, como también he intentado mostrar en estas páginas, prosistas y dramaturgos del Siglo de Oro, entre los que se cuentan a Lope de Vega, Sebastián Mey, Castillo Solórzano o Juan Pérez de Montalbán, leyeron la antología de Francesco Sansovino y recrearon algunas de sus historias.

\section{BIBLIOGRAFÍA}

Berruezo Sánchez, Diana (2012): "Il Novellino de Masuccio Salernitano en algunas comedias de Lope y Calderón”, en Sònia Boadas, Félix Ernesto Chávez y Daniel García Vicens (eds.), La tinta en la clepsidra. Fuentes, historia y tradición en la literatura hispánica, Barcelona, PPU, pp. 139-149.

Berruezo Sánchez, Diana (2013): "Entre alcahuetas anda el juego. La francesilla de Lope de Vega y dos novelas de Masuccio Salernitano", en Alain Bègue y Emma Herrán Alonso (dirs.), Pictavia aurea. Actas del IX Congreso de la Asociación Internacional "Siglo de Oro", Toulouse, Presses Universitaires du Mirail, pp. 747-754.

Berruezo Sánchez, Diana (2014): "Il Novellino” de Masuccio Salernitano y su influencia en la literatura de la Edad de Oro, tesis doctoral, Barcelona, Universidad de Barcelona.

Berruezo Sánchez, Diana (2015): Il Novellino de Masuccio Salernitano y su influencia en la literatura española de la Edad de Oro, Vigo, Academia del Hispanismo.

Berruezo Sánchez, Diana (en prensa): "La antología de Francesco Sansovino y sus novellieri ante la censura: los casos de Giovanni Boccaccio y Masuccio Salernitano”, en Víctor Lillo, Dámaris Montes y M. José Vega (eds.), Saberes inestables. Estudios sobre censura y expurgación en la España de los siglos XVI y XVII, Madrid/Frankfurt am Main, Iberoamericana/Vervuert.

Boccaccio, Giovanni (1551): Il Filocopo di M. Giovanni Boccaccio. Di nuovo riveduto, corretto, et alla sua vera lettione ridotto da M. Francesco Sansovino. Con la Tavola di tutte le materie che nell'opera si contengono, Venezia, Giovita Rampiro.

Boccaccio, Giovanni (1554): Il Filocopo di M. Giovanni Boccaccio. Di nuovo riveduto, corretto, et alla sua vera lettione ridotto da M. Francesco Sansovino. Con la Tavola di tutte le materie che nell'opera si contengono, Venezia, Francesco Rampazetto. 
Boccaccio, Giovanni (1564): Il Filocopo di M. Giovanni Boccaccio. Di nuovo riveduto, corretto, et alla sua vera lettione ridotto da M. Francesco Sansovino. Con la Tavola di tutte le mateire che nell'opera si contengono, Venezia, Francesco Lorenzini.

Boccaccio, Giovanni (1575): Il Filocopo di M. Giovanni Boccaccio. Di nuovo riveduto, corretto, et alla sua vera lettione ridotto da M. Francesco Sansovino. Con la Tavola di tutte le mateire che nell'opera si contengono, Venezia, Antonio Bertano.

Boccaccio, Giovanni (1612): Il Filocopo di M. Giovanni Boccaccio. Di nuovo riveduto, corretto, et alla sua vera lettione ridotto da M. Francesco Sansovino. Con la Tavola di tutte le materie che nell'opera si contengono, Venezia, Lucio Spineda.

Bonora, Elena (1994): Ricerche su Francesco Sansovino. Imprenditore librario e letterato, Venezia, Istituto Veneto di Scienze, Lettere ed Arti.

Bottasso, Enzo (1989): "La prima circolazione a stampa", en Stefano Bianchi (ed.), La novella italiana. Atti del Convegno di Caprarola. 19-24 settembre 1988, Roma, Salerno editrice, t. 1, pp. 245-264.

Bourland, Caroline B. (1973 [1927]): The short story in Spain in the Seventeenth Century with a bibliography of the novella from 1576 to 1700, New York, Burt Franklin.

Bravo-Villasante, Carmen (1975): "Introducción", en Sebastián Mey, Fabulario, Madrid, Fundación Universitaria Española, pp. 8-21

Buchanan, Milton A. (1906a): "Sebastián Mey's Fabulario I", Modern Language Notes, 21-6, pp. 167-171.

Buchanan, Milton A. (1906b): "Sebastián Mey’s Fabulario II", Modern Language Notes, 21-7, pp. 201-205.

Castillo Solórzano, Alonso de (1649): La quinta de Laura, que contiene seis novelas adornadas de diferentes versos por don Alonso de Castillo Solórzano, Zaragoza, Real Hospital de nuestra señora de Gracia a costa de Matías de Lizán.

Castillo Solórzano, Alonso de (1985): Las harpías en Madrid, Pablo Jauralde (ed.), Madrid, Castalia.

Cicogna, Emmanuele Antonio (1834): Delle iscrizioni veneziane raccolte ed illustrate da Emmanuele Antonio Cicogna. Cittadino veneto, vol. IV, Venezia, Giuseppe Picotti Stampatore.

Di Filippo Bareggi, Claudia (1988): Il mestiere di scrivere. Lavoro intellettuale e mercato librario a Venezia nel Cinquecento, Roma, Bulzoni editore.

Dixon, Victor (1983): "La mayor confusión", Hispania, 3, pp. 17-28.

Fernández Rodríguez, Daniel (2016a): "Una fuente olvidada del Guzmán de Alfarache: la novella de «Due Giovani Sanesi» de Parabosco (y unas notas sobre Masuccio, Sansovino y Tamariz)", Edad de Oro, XXV, pp. 175-190.

Fernández Rodríguez, Daniel (2016b): "La influencia de las novelas de Girolamo Parabosco (pasando por Sansovino) en la literatura española del Siglo de Oro", Estudios Románicos, 25, pp. 217-228.

Fernández Rodríguez, Daniel (2016c): "La difusión y recepción de las novelas de Agnolo Firenzuola en el Siglo de Oro", en Michela Graziani y Salomé Vuelta García (eds.), Traduzioni, riscritture, ibridazioni. Prosa e teatro fra Italia, Spagna e Portogallo, Florencia, Leo Olschi.

Gasparetti, Antonio (1939): Las "Novelas" de Mateo María Bandello como fuentes del teatro de Lope de Vega Carpio, Salamanca, Imp. Cervantes.

Giuliani, Luigi (1992): "Introducción", en Juan Pérez de Montalbán, Sucesos y prodigios de amor, Barcelona, Montesinos, pp. 7-55.

González Ramírez, David (2011), "En el origen de la novela corta del Siglo de Oro: los novellieri en España", Arbor, 752, pp. 1221-1243.

González Ramírez, David (2012): "En el origen de la novela corta del Siglo de Oro: los novellieri desde sus paratextos", Arbor, 756, pp. 813-828.

González Ramírez, David e Ilaria Resta (2013): "Traducción y reescritura en el Siglo de Oro: L'ore di ricreazione de Ludovico Guicciardini en España”, en Isabel Colón Calderón, David Caro Bragado, Clara Marías y Alberto Rodríguez (coords.), Pampinea y sus descendientes: novella y novela española en los Siglos de Oro, Madrid, Sial, pp. 61-76. 
Helí Hernández, Jesús (1982): Antecedentes italianos de la novela picaresca española: aspectos literarios y lingüísticos, Madrid, José Porrúa.

Jauralde Pou, Pablo (1985): "Introducción" y notas a Alonso de Castillo Solórzano, Las harpías en Madrid, Pablo Jauralde (ed.), Madrid, Castalia.

Krenkel, Max (1887): Klassiche Bühnendichtungen der Spanier, III. Calderon. Der Richter von Zalamea, Leipzig, Johann Ambrosius Barth.

Malato, Enrico (1989): "La nascita della novella italiana: un'alternativa letteraria borghese alla tradizione borghese", en Stefano Bianchi (ed.), La novella italiana. Atti del Convegno di Caprarola. 19-24 settembre 1988, Roma, Salerno Editrice, t. 1, pp. 3-45.

Masuccio Salernitano (2000): Il Novellino nell'edizione di Luigi Settembrini, Silvano Nigro (ed.), Milano, RCS Libri.

Menéndez Pelayo, Marcelino (1969): “Observaciones preliminares”, en Obras de Lope de Vega, Madrid, BAE, t. XXV, pp. 155-169.

Mey, Sebastián (1975): Fabulario, Carmen Bravo-Villasante (ed.), Madrid, Fundación Universitaria Española.

McGrady, Donald (1981): "Introducción”, en Lope de Vega, La francesilla, Charlottesville, Biblioteca del Siglo de Oro, pp. 3-60.

Molina, Julián (2002): "Prólogo", en Lope de Vega, El galán Castrucho, en Comedias de Lope de Vega. Parte IV, Lérida, Milenio, t. 3, pp. 1087-1102.

Muñiz Muñiz, M. ${ }^{a}$ de las Nieves (dir.) (2002-2009): Proyecto Boscán: Catálogo de las traducciones españolas de obras italianas (hasta 1939), [Barcelona, Universidad de Barcelona/Scuola Normale Superiore di Pisa/Istituto Italiano di Cultura de Barcelona], <http://www.ub.edu/ boscan>.

Ordine, Nuccio (2009): "Francesco Sansovino da Un discorso fatto sopra il Decamerone [1571]", en Teoria della novella e teoria del riso nel Cinquecento, Napoli, Liguori, pp. 183-188.

Passano, Giambattista (1965 [1878]): I novellieri italiani in prosa, I, Bologna, Arnaldo Forni Editore.

Pérez de Montalbán, Juan (1992): Sucesos y prodigios de amor, Luigi Giuliani (ed.), Barcelona, Montesinos.

Pirovano, Donato (1996): "Masuccio e la critica", Giornale storico della letteratura italiana, 173, 563, pp. 392-428.

Plaisance, Michel (1989): "Funzione e tipologia della cornice", en Stefano Bianchi (ed.), La novella italiana. Atti del Convegno di Caprarola. 19-24 settembre 1988, Roma, Salerno editrice, t. 1, pp. 103-118.

Roaf, Christina (1993): "Cultura e conoscenze di un giovane del Cinquecento: Francesco Sansovino e le Lettere sopra le dieci giornate del Decamerone", en Omaggio a Gianfranco Folena, Padova, Editoriale Programma, vol. 2, pp. 1107-1122.

Rojo Vega, Anastasio (1994): "Manuscritos y problemas de edición en el siglo XVI", Castilla, 19, pp. 129-157.

Salas Barbadillo, Alonso Jerónimo de (1985): La ingeniosa Elena, Jesús Costa Ferrandis (ed.), Lérida, Instituto de Estudios Ilerdenses.

Sansovino, Francesco (1561): Cento novelle scelte dai più nobili scrittori, per Fran. Sansovino. Nelle quali piacevoli et aspri casi d'Amore, et altri notabili avenimenti si leggono. Con privilegio, Venezia, Sansovino.

Sansovino, Francesco (1562): Le cento novelle scelte da più nobili scrittori della lingua volgare di Francesco Sansovino. Nelle quali piacevoli et aspri casi d'Amore, et altri notabili avvenimenti si leggono. Di nuovo ampliate, riformate, rivedute et corrette per il medesimo. Con privilegio per Anni $\mathrm{XV}$, Venezia, [s. e.].

Sansovino, Francesco (1563): Delle cento novelle scelte da più nobili scrittori della lingua volgare di M. Francesco Sansovino. Editione terza. Nelle quali piacevoli et aspri casi d'Amore, et altri notabili avvenimenti si leggono. Di nuovo ampliate, riformate, rivedute et corrette per il medesimo, 
Venezia, Fr. Rampazetto, <http:/www.mdz-nbn-resolving.de/urn/resolver.pl?urn=urn:nbn:de: bvb:12-bsb10190099-2>.

Sansovino, Francesco (1566): Cento novelle scelte da più nobili scrittori della lingua volgare di Francesco Sansovino. Nelle quali piacevoli, et aspri casi d'amore, et altri notabili avvenimenti si contengono. Di nuovo ampliate, reformate, rivedute, et corrette, per il medesimo, et aggiontovi di nuovo le figure in principio d'ogni Novella. Con privilegio per anni xv, Venezia, [Fr. Sansovino].

Sansovino, Francesco (1571): Cento novelle scelte da più nobili scrittori della lingua volgare, con l'aggiunta di cento altre novelle antiche non pur belle per inventione, ma molto vili per l'eleganti et toscae elocutioni necessarie a chi vuol regolatamente scrivere nella nostra lingua. Nelle quali piacevoli, et aspri casi d'Amore, et altri notabili avvenimenti si contengono. Con gli argomenti a ciascuna novella per ammaestramento de'Lettori al viver bene. Et con le figure poste et apropriate a suoi luoghi. Di nuovo rifecute, corrette et riformate in questa Quarta Impressione. Con privilegio, Venezia, Marchiò Sessa.

Sansovino, Francesco (1598): Cento novelle scelte da più nobili scrittori della lingua volgare de Franscesco Sansovino, nelle quali piacevoli et notabili avvenimenti si contengono. Di nuovo reformate, rivedute, et corrette, con licentia de' superiroi et aggiunotovi le Figure in principio d'ogni Novella. All'Illustiss. Sig. Annibal Chieppi, consigliero del serenissimo Duca di Mantova. Con privilegio, Venezia, alla stampa di Marco Claseri, appresso Alessandro de Vecchi.

Sansovino, Francesco (1603): Cento novelle scelte da piu nobili scrittori della Lingua Volgare, di Francesco Sansovino: Nelle quali piacevoli, e notabili avvenimenti si contengono: di nuovo reformate, rivedute, et corrette, con licentia de' Superiori; e aggiuntovi novamentele figure in principio d'ogni Novella. Al Magnifico, et Eccellente Signore e padron colendissimo, il Sig. Girolamo Rossetti. Con privilegio, Venezia, De Vecchi.

Sansovino, Francesco (1610): Cento novelle scelte da' più nobili scrittori della Lingua Volgare, di Francesco Sansovino: Nelle quali si contengono piacevoli, e notabili avvenimenti. Nuovamente reformate, rivedute, et corrette, con licenza de' Superiori; e di nuovo aggiontovi le figure ad ogni Novella. Al Magnifico, et Eccelente Signore e padron colendissimo, il Sig. Girolamo Rossetti, Venezia, De Vecchi.

Sansovino, Francesco (1942), "L'avvocato" e "Il segretario", Piero Calamandrei (ed.), Firenze, Le Monnier.

Terrusi, Leonardo (1998): Il Novellino di Masuccio Salernitano. Revisioni tipografiche e ricezione linguistica quattro-cinquecentesca. Tesi di dottorato di ricerca. Anno accademico 1997-98. Coordinatore: Prof. Michele Dell'Acquila. Tutore: Prof. Ruggiero Stefanelli. Università degli Studi di Bari. Dottorato di ricerca in Disciplinte linguistiche, filologiche e letterarie, vol I y II, [s. 1.], [s. e.].

Vega, Lope de (1620): Trezena parte de las comedias de Lope de Vega Carpio, Madrid, Viuda de Alonso Martín.

Fecha de recepción: 27 de junio de 2015

Fecha de aceptación: 15 de septiembre de 2015 